\title{
SELFDUALITIES OF SERIAL RINGS, REVISITED
}

\author{
PHAM NGOC ÁNH
}

\begin{abstract}
A description is given of serial rings whose maximal quotient rings are quasi-Frobenius (QF). Every serial ring is a factor of a serial ring whose maximal quotient ring is a QF-ring. This result is used to give a new, conceptual proof for the selfduality of serial rings emphasising the importance of weakly symmetric rings.
\end{abstract}

\section{INTRODUCTION}

The question as to whether serial rings are selfdual was put by Haack [5], and was answered in positive by Dischinger and Müller [4]. Waschbüsch [16] noticed that the result had been claimed (without proof) earlier by Amdal and Ringdal ([2] Remark $5(\mathrm{c}))$. In [16] he presents a proof which uses Kupisch's classification of serial rings described in [1], [2], [10]. All these proofs, however, are of highly technical nature; furthermore, using the Kupisch classification for this purpose seems to us like using a sledge hammer to crack an almond. It is therefore quite reasonable to look for a conceptual proof, and it is not surprising that several authors (see e.g. [6], [7], [8], $\left.[9],[13],[14]^{1}\right)$ are still working on this fascinating topic.

1991 Mathematics Subject Classification. Primary 16D90, 16D50; Secondary 16D10 .

Key words and phrases. Endomorphism ring, automorphism, serial ring, quasi-Frobenius.

This research was supported partly by Hungarian National Foundation for Scientific Research grant no. T34530 and T43034. It is also supported by a German-Hungarian exchange program .

Much of the work which led to the results contained in this article was completed while the author was a Visiting Professor at University of Iowa during Spring 2001. Thanks to the university, and especially to Professor Kent Fuller, for their support.

${ }^{1}$ The author is indebted to the referee for calling his attention to the works [7], [13] and [14] where Theorem 3.5 is also proved. 
In this paper we present such a conceptual proof. We describe serial rings as factors of serial rings whose maximal quotient rings are quasi-Frobenius (QF), and then show that the latter rings admit weakly symmetric selfduality. By an observation of Haack, however, such selfdualities carry over to factor rings.

\section{BASIC FACTS, NOTATIONS}

For the benefit of the reader we present some easy, but basic results and notation from [3], [10] in such an order that their proofs can be easily deduced.

The radical, the length and the injective hull of a module $M$ is denoted by $J(M)$, $c(M)$ and $I(M)$, respectively, and module homomorphisms will be written opposite the scalars. $J$ will be the radical of a ring $R$. An artinian $\operatorname{ring} R$ is called selfdual if there is a ring isomorphism $\varphi: R \longrightarrow \operatorname{End}(E)$ for some injective cogenerator ${ }_{R} E$. Note that $E$ is in general not $I(R / J)$. The question of selfduality is probably the most intriguing puzzle in the theory of Morita duality. It turns out that even for the class of serial rings - the best-understood class of non-semisimple rings - it is not simple to check a selfduality. Since minimal injective cogenerators are quite complicated, with a few exceptions when a ring is commutative or hereditary with some additional properties, it is not an easy job to find out a way of embedding a ring into the endomorphism ring of an appropriate injective cogenerator. The isomorphism $\varphi$ induces a weakly symmetric selfduality if $E \varphi(e) \cong I(R e / J e)$ for all $e^{2}=e \in R$. In particular, a QF-ring $R$ is called weakly symmetric if $R e \cong$ $I(R e / J e)$ for every $e^{2}=e \in R$. A selfduality $\varphi$ is called a $\operatorname{good}$ duality if $\varphi(K)=$ $r_{R} r_{E}(K)$ for every ideal $K$ of $R$ where $r_{X}(Y)$ denotes the right annihilator of $Y$ in $X$ with respect to the multiplication under consideration. A good duality obviously induces selfdualities for factor rings. A serial ring is an artinian ring over which each module is a direct sum of uniserial modules (that is, modules with chain for subomdule lattices). Avoiding triviality we will consider only serial rings which are not uniserial: that is not local ones.

In what follows, with one exception in Proposition 4.1, $R$ is an indecomposable, basic, serial ring with a basic set $\left\{e_{i} \mid i=1, \ldots, n\right\}$ of idempotents such that there 
are projective covers $R e_{i-1} \longrightarrow J e_{i}$ for $i=2, \ldots, n$ and $R e_{n} \longrightarrow J e_{1}$ for the case $J e_{1} \neq 0$. This means that the associated quiver of $R$ is $A_{n}$ for the case $J e_{1}=0$ or $\tilde{A}_{n-1}$ for $J e_{1} \neq 0$. Let $[k]$ be the least positive integer congruent to $k \in \mathbb{Z}$ modulo $n$ and

$$
S_{i}=R e_{i} / J e_{i}, P_{i}=R e_{i}, \quad R_{i}=e_{i} R e_{i}, \quad c_{i}=c\left({ }_{R} P_{i}\right) .
$$

Fix $a_{i} \in e_{i} R e_{[i+1]} \backslash J^{2}(i=1, \ldots, n)$ with $a_{n}=0$ in case $J e_{1}=0$ and for $k \in \mathbb{N}$ let $\stackrel{k}{a}{ }_{i}:=a_{[i-k+1]} \ldots a_{[i-1]} a_{i}$. Then we have $\stackrel{1}{a}_{i}=a_{i}$ and

$$
\stackrel{n}{a}_{[i-1]} \in R_{i}, \stackrel{n}{a}_{i} \in R_{[i+1]}, \stackrel{n}{a}_{[i-1]} a_{i}=\stackrel{n+1}{a}_{i}=a_{i} \stackrel{n}{a}_{i}, \quad R \stackrel{k}{a_{i}}=J^{k} e_{[i+1]}
$$

and

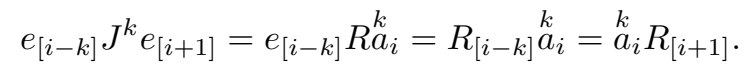

If, starting from the top, $N_{1}, N_{2}, \ldots, N_{c}(c=c(M))$ are the composition factors of a uniserial module $M$, then

$$
N_{i} \cong N_{j} \Longleftrightarrow[i]=[j] .
$$

Since a simple $R$-module $S$ is isomorphic to $S_{k}$ iff $e_{k} S \neq 0$, we obtain

$$
e_{k} J^{j} e_{i} \neq e_{k} J^{j+1} e_{i} \Longleftrightarrow[i-j]=k, c_{i}>j .
$$

Observing that the proof of implication (d) $\Longrightarrow$ (a) in Theorem 32.2 in [3] works also for semiprimary rings, we get

Proposition 2.1. A semiprimary ring $R$ is serial iff $R / J^{2}$ is serial.

Proposition 2.2. Every serial QF-ring $R$ admits a weakly symmetric selfduality $\Phi: R \longrightarrow R$ such that $\Phi\left(e_{i}\right)=e_{[i+1-c]}$ where $c=c\left(P_{i}\right)(i=1, \ldots, n)$.

Proof. By assumption all $c_{i}$ are equal, say, to $c$. Then $[c]=1$ if and only if $R$ is weakly symmetric by (3). Therefore it is sufficient to prove the case $[c] \geq 2$. This implies $1 \leq[c]-1=[c-1]$. The length of the module $R_{i} e_{i} R e_{[i+1]}$ is the number of the simple factors of ${ }_{R} P_{[i+1]}$ isomorphic to $S_{i}$ which is precisely $l+1$ if $l$ is the greatest positive integer satisfying $l n \leq c-1$ by (3). Therefore, as 
$1 \leq[c]-1=[c-1]$ and again in view of (3), $l+1$ is also the length of $R_{[i+1]} R_{[i+1]}$ which is the number of simple factors of $P_{[i+1]}$ isomorphic to $S_{[i+1]}$. Similarly, one obtains that the length of the right $R_{[i+1]}$-module $e_{i} R e_{[i+1]}$ is equal to the length of ${ }_{R_{i}} R_{i}$. Since the lengths of the uniserial right $R_{[i+1]}$-module $e_{i} R e_{[i+1]}$ and the left $R_{i}$-module $e_{i} R e_{[i+1]}$ are equal, we see that $e_{i} R e_{[i+1]}$ is cyclic and free both as a left $R_{i}$ - and a right $R_{[i+1]}$-module. Consequently the $a_{i}$ induce ring isomorphisms

$$
g_{i}: R_{[i+1]} \longrightarrow R_{i}: x \in R_{[i+1]} \mapsto y \in R_{i}: y a_{i}=a_{i} x
$$

and

$$
\stackrel{k}{g}_{i}=g_{[i-k+1]} \cdots g_{[i-1]} g_{i}: R_{[i+1]} \longrightarrow R_{[i-k+1]}, \quad k \in \mathbb{N} .
$$

Thus $\stackrel{1}{g}_{i}=g_{i}$ and in view of (1) we have

$$
g_{i}\left(\stackrel{n}{a}{ }_{i}\right)=\stackrel{n}{a}_{[i-1]}, \quad g_{i}\left(\stackrel{n k}{a_{i}}\right)=\stackrel{n k}{a}_{[i-1]} \quad(i=1, \ldots, n ; k \in \mathbb{N})
$$

We construct an automorphism $\varphi$ of $R$ satisfying $\varphi\left(e_{i}\right)=e_{[i-1]}$ as follows. If $x \in R_{i}$, put $\varphi(x)=g_{[i-1]}(x) \in R_{[i-1]}$. For $z \in e_{k} J^{p} e_{i}$ one can assume in view of (2) and (4) that

$$
[i-p]=k, \quad z=\stackrel{p}{a}_{[i-1]}, \quad x \in R_{k}=R_{[i-p]}
$$

and put

$$
\varphi(z)=\varphi\left(\stackrel{p}{x}_{[i-1]}^{p}\right)=g_{[k-1]}(x) \stackrel{p}{a}{ }_{[i-2]} \in e_{[k-1]} R e_{[i-1]} .
$$

$\varphi$ is well-defined on $e_{k} R e_{i}$. For if $z \in e_{k} J^{q} e_{i}, \quad[i-q]=k, z=y_{[a}^{q}{ }_{[i-1]}$ and $t$ is the smallest positive integer with

$$
J^{t-1} a_{i} / J^{t} a_{i}=R_{a}^{t} a_{i} / R_{a}^{t+1}{ }_{i} \cong S_{k},
$$

then $p-t=n n_{1}, q-t=n n_{2}$ for some nonnegative integers $n_{1}, n_{2}$ and

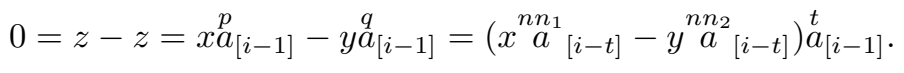

This implies together with (5)

$$
\begin{aligned}
& 0=g_{[k-1]}\left(x \stackrel{n n_{1}}{a}{ }_{[i-t]}-y \stackrel{n n_{2}}{a}{ }_{[i-t]}\right) \stackrel{t}{a}_{[i-2]}=g_{[k-1]}(x)^{n n_{1}}{ }_{[i-t-1]}^{t} \stackrel{t}{[i-2]}^{-}
\end{aligned}
$$

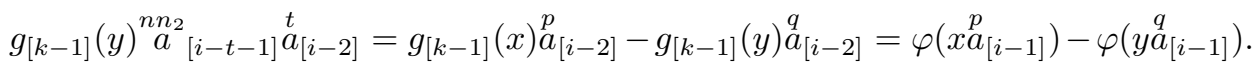


Since $c\left(R_{[i-1]} e_{[i-1]} R e_{[j-1]}\right)=c\left(R_{i} e_{i} R e_{j}\right)$ by (3), $\varphi$ is an additive isomorphism between $e_{[i-1]} R e_{[j-1]}$ and $e_{i} R e_{j}$ for all $i, j=1, \ldots, n$. Therefore $\varphi$ can be extended to an additive automorphism of $R$. In this case $\varphi$ is also multiplicative, by which we mean $\varphi(u w)=\varphi(u) \varphi(w)$ for any $u, v \in R$. It obviously suffices to see $\varphi(u w)=\varphi(u) \varphi(w)$ for any $u=e_{q} u e_{k}$ and $w=e_{k} w e_{i}(q, k, i=1, \ldots n)$. Write

$$
u=\stackrel{j}{a}_{[k-1]}, \quad w=\stackrel{p}{a}_{[i-1]} ; \quad x \in R_{q}, \quad y \in R_{k}, \quad[k-j]=q, \quad[i-p]=k,
$$

then

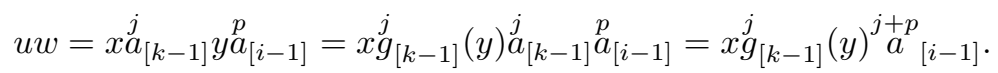

Thus we have

$$
\begin{gathered}
\varphi(u w)=g_{[q-1]}\left(x^{j} g_{[k-1]}(y)\right)^{j+p} a_{[i-2]}=g_{[q-1]}(x) g_{[q-1]}\left(g_{[k-1]}^{j}(y)\right)^{j+p}{ }_{[i-2]}= \\
g_{[q-1]}(x)^{j+1}{ }_{[k-1]}(y) \stackrel{p}{a}_{[k-2]}^{j} \stackrel{p}{[i-2]}^{p}=g_{[q-1]}(x) \stackrel{j}{a}_{[k-2]} g_{[k-1]}(y) \stackrel{p}{a}_{[i-2]}^{p}=\varphi(u) \varphi(w) .
\end{gathered}
$$

Let $l=[c-1]=[c]-1$ and $\Phi=\varphi^{l}$. If we defines an $R$ - $R$-bimodule $R_{\Phi}$ by $R_{\Phi}:=R, r * x * t=r x \Phi(t) \quad(r, x, t \in R)$, then $R_{\Phi}$ induces a weakly symmetric selfduality for $R$ because the socle of $R_{\Phi} * e_{i}=R \Phi\left(e_{i}\right)=R e_{[i+1-[c]]}$ is exactly $S_{[i+c-[c]]}=S_{i}$ for all $i=1, \ldots, n$.

Remark 2.0.1. This result was proved by Haack (cf. Theorem 3.3 [5]) though it is also observed earlier by Kupisch (cf. footnote 4 [10]) as a consequence of his classification. The above proof is a simplified version of the much easier proof to Satz 2.1 [10]. Indeed, both proofs are based implicitly on the fact that the $\stackrel{k}{a} a_{i}$ together with the idempotents $1,0, e_{1}, \ldots, e_{n}$ form a semigroup under multiplication. Roughly speaking, this semigroup can be considered as a "multiplicative base" or in the other words, a "Cartan basis", of a serial ring in a generalized sense that the corresponding images yield a basis of the graded ring associated to the filtration given by powers of the radical. Moreover, in the case of a not weakly symmetric serial QF-ring the permutation sending $i$ to $[i-1]$ induces an automorphism of this multiplicative semigroup which can be extended to a ring automorphism. We do 
not know about the existence of such a "multiplicative basis" for locally distributive rings and this lack of knowledge of existence is probably also a reason why a corresponding question on selfduality for such rings or even for a narrower class of regular representation-finite rings seems to be more difficult. The advantage of Haack's proof is a quite natural, easily understandable definition of the map $\varphi$. This map $\varphi$ in Haack's proof is immediately multiplicative by observing the obvious equality $a_{i} x=\varphi(x) a_{j}$ for all $x \in e_{[i+1]} R e_{[j+1]}$. Furthermore, Proposition 2.2 is equivalent to the statement that the automorphism group of a serial, not weakly symmetric QF-ring contains a cyclic subgroup of order $n$.

\section{StruCture OF SERIAL RINGS}

Let $P_{l_{1}}, \ldots, P_{l_{m}}\left(1 \leq l_{1}<l_{2}<\cdots<l_{m} \leq n\right)$ be the injective indecomposable projectives. Let $l_{0}=0$ and $\{k\}$ be the least positive integer congruent to $k$ modulo $m$ for each $0 \neq k \in \mathbb{Z}$. Moreover, define $\{0\}=0$ if $J e_{1}=0$, otherwise $\{0\}=m . P_{l_{k}}$ has exactly $\delta_{k}$ nonzero projective submodules where $\delta_{1}$ is $l_{1}$ if $J e_{1}=0$ or $n-l_{m}+l_{1}$ if $J e_{1} \neq 0$, and $\delta_{k}=l_{k}-l_{\{k-1\}}(k=2, \ldots, m)$. Let

$$
e=e_{l_{1}}+\cdots+e_{l_{m}}, \quad I_{i}=I\left(P_{i}\right), \quad I=I\left({ }_{R} R\right)=\bigoplus_{i=1}^{n} I_{i} \cong \bigoplus_{k=1}^{m} P_{l_{k}}^{\delta_{k}}, \quad T=\operatorname{End}\left({ }_{R} I\right) .
$$

Here, ${ }_{R} R e$ is a minimal faithful left ideal (that is, a direct sum of isomorphism types of indecomposable injective projectives in the case of serial rings), and $T$ is a serial ring as it is Morita equivalent to $e R e$. Let $\varepsilon_{i}$ be the projection of $I$ onto $I_{i}$ and $T_{i}=T \varepsilon_{i}(i=1, \ldots, n)$. Put

$$
B=\{t \in T \quad \mid R t=0\}, \quad A=\{t \in T \quad \mid R t \subseteq R\} .
$$

Proposition 3.1. If $B=0$, then $T$ is a QF-ring.

Proof. Having $B=0$ implies that $R$ can be identified as a subring of $T$ in the usual way. Since $a_{\left[l_{k}+i\right]}\left(1 \leq i \leq \delta_{\{k+1\}}-1 ; k=1, \ldots, m\right)$ induces an isomorphism between ${ }_{T} T \varepsilon_{\left[l_{k}+i\right]}$ and ${ }_{T} T \varepsilon_{\left[l_{k}+i+1\right]}$, all ${ }_{T} T \varepsilon_{\left[l_{k}+1\right]}, \ldots,{ }_{T} T \varepsilon_{l_{\{k+1\}}}$ are isomorphic and ${ }_{T} T a_{l_{k}}$ is the radical of ${ }_{T} T \varepsilon_{\left[l_{k}+1\right]}$. Observing that the kernel of $a_{l_{k}}$ is not zero, we obtain that ${ }_{T} T \varepsilon_{l_{k}}$ is injective for all $k=1, \ldots, m$, i.e. $T$ is a QF-ring. 
Remark 3.0.2. It is obvious that $B=0$ if and only if the socle of ${ }_{R} R$ is a sum of simple modules $S_{i}\left(i \in\left\{\left[l_{k}+1\right] \mid k=1, \ldots, m\right\}\right)$ or equivalently if the $e_{i} R_{R}$ $\left(i \in\left\{\left[l_{k}+1\right] \mid k=1, \ldots, m\right\}\right)$ are the indecomposable projective injectives. Moreover, $B=0$ implies $t \in e_{i} R e_{j}$ if $t \in e_{i} T e_{j}$ and $P_{i} t \subseteq P_{j}$. Consequently, $e_{i} R e_{j}=e_{i} T e_{j}$ if $P_{j}$ is not isomorphic to a submodule of $P_{i}$. If $P_{j}$ is isomorphic to a submodule of $P_{i}$, then $P_{i} t \subseteq P_{j}$ for all $t \in e_{i} T e_{j}$ which are not isomorphisms between $I_{i}$ and $I_{j}$, i.e., $e_{i} R e_{j}$ is precisely the radical of ${ }_{R_{i}} e_{i} T e_{j_{R_{j}}}$. Therefore for the sum $f_{k}$ of idempotents $e_{i}$ such that $P_{i}$ is isomorphic to submodule of $P_{l_{k}}, f_{k} R f_{k}=\operatorname{End}\left(R f_{k}\right)$ is a $\delta_{k} \times \delta_{k}$ matrix ring of the form

$$
\left(\begin{array}{ccccc}
S & S & \ldots & S & S \\
M & S & \ldots & S & S \\
\vdots & \ddots & \ddots & \vdots & \vdots \\
M & M & \ddots & S & S \\
M & M & \ldots & M & S
\end{array}\right)
$$

where $S=R_{l_{k}}$ and $M$ is its radical. Thus serial rings with $B=0$ are a common generalization of both serial QF-rings and the so-called $(S: M)$-upper triangular matrix rings that appear in the characterization of semiperfect HNP rings. Moreover, $B=0$ implies also $R e_{l_{k}}=T e_{l_{k}}(k=1, \ldots, m)$. For if $t=t e_{l_{k}}$, then $I t \subseteq R$ and thus its restriction to $R$ is some $r \in \operatorname{Re}_{l_{k}}$, hence $t=r \in \operatorname{Re}_{l_{k}}$.

Theorem 3.2. $B=0$ if and only if the maximal quotient ring of $R$ is a QF-ring.

Proof. If $B=0$, then $R$ is a subring of $T$ and ${ }_{R} T=I=I\left({ }_{R} R\right)$ by the previous remark. Hence $T$ is the biendomorphism ring of $I$, i.e., the maximal quotient ring of $R$ by its definition given in [12]. Thus the maximal quotient ring of $R$ is a QF-ring by Proposition 3.1.

Conversely, if the maximal quotient ring of $R$ is a QF-ring, then $T$ must be the maximal quotient ring of $R$ and hence $B=0$ in view of [12] Propositions 4.3.2, 4.3.3 and 4.3.6. 
Remark 3.0.3. For an arbitrary ring $R$ the equality $B=0$ holds if an only if the maximal left quotient ring is left selfinjective. For, $B=0$ means that $I_{T}=1 T$ is a free right $T$-module and hence $I=T=\operatorname{End}\left(I_{T}\right)$. If the maximal quotient ring of $R$ is $\mathrm{QF}$, then the endomorphism ring of a minimal faithful $R$-module is obviously also QF, but the converse is not true. For example, if $R$ has a strictly increasing admissible sequence $\left\{c_{1}, \ldots, c_{n}\right\}(n>1)$ such that $\left[c_{1}\right] \neq 1$, then ${ }_{R} R e_{n}$ is the minimal faithful module and $R_{n}=e_{n} R e_{n}$ is trivially $\mathrm{QF}$, but $B \neq 0$, i.e., the maximal quotient ring of $R$ cannot be QF.

We can characterize serial rings $R$ with $B=0$ as follows

Theorem 3.3. For each $k=1, \ldots, m$ and $i=1, \ldots, \delta_{k}$, let

$$
W_{k}^{i}:=\bigoplus_{l=\left[l_{\{k-1\}}+i\right]}^{l_{k}} T_{l}, \quad W_{k}=W_{k}^{1}, \quad \widetilde{W}_{k}^{i}=W_{k}^{i} \oplus \bigoplus_{l=\left[l_{\{k-1\}}+1\right]}^{\left[l_{\{k-1\}}+i-1\right]} J\left(T_{T} T_{l}\right)
$$

If $B=0$, then $R$ is the ring of all endomorphisms of ${ }_{T} T$ satisfying $W_{k}^{i} r \subseteq \widetilde{W}_{k}^{i}$ for all $i \in\left\{1, \ldots, \delta_{k}\right\}$ provided $r$ induces an endomorphism of $W_{k}(k=1, \ldots, m)$.

Conversely, let $T$ be a serial QF-ring with a basic set $\left\{e_{i} \mid i=1, \ldots, n\right\}$ of idempotents and $e=e_{l_{1}}+\cdots+e_{l_{m}}\left(1 \leq l_{1}<l_{2}<\cdots<l_{m} \leq n\right)$ be such that for $k=1, \ldots, m_{T} T_{i} \cong_{T} T_{l_{k}}\left(i=\left[l_{\{k-1\}}+1\right], \ldots, l_{k}\right)$. Let $R$ be the ring of all endomorphisms $r$ of ${ }_{T} T$ satisfying $W_{k}^{i} r \subseteq \widetilde{W}_{k}^{i}$ if $r$ induces an endomorphism of $W_{k}$. Then $R$ is a serial ring with $T=\operatorname{End}\left(I\left({ }_{R} R\right)\right)$ and $B=0$.

Proof. The first statement of this theorem is already proved in Remark 3.0.2.

For the second statement it is clear that $e_{i} R e_{j}=e_{i} T e_{j}$ if ${ }_{T} T e_{i}$ and ${ }_{T} T e_{j}$ are nonisomorphic. Moreover, both ${ }_{R} R e_{i}$ and $e_{i} R_{R}\left(i=l_{[\{k-1\}+1]}, \ldots, l_{k}\right)$ are isomorphic to submodules of ${ }_{R} R e_{l_{k}}$ and $e_{[l\{k-1\}+1]} R_{R}$, respectively. Therefore simple $T$-modules $T e_{l_{k}} / J(T) e_{l_{k}}$ and $e_{l_{k}} T / e_{l_{k}} J(T)(k=1, \ldots, m)$ are uniserial $R$-modules with socles $R e_{\left[l_{\{k-1\}}+1\right]} / J e_{\left[l_{\{k-1\}}+1\right]}$ and $e_{l_{k}} R / e_{l_{k}} J$, respectively. These facts altogether imply that ${ }_{R} R e_{i}$ and $e_{i} R_{R}(i=1, \ldots, n)$ are uniserial and hence $R$ is a serial ring. It is now routine to check that $B=0$ and $T=\operatorname{End}\left(I\left({ }_{R} R\right)\right)$. 
Remark 3.0.4. Theorem 3.3 shows that in the case $B=0$ there is a one-to-one correspondence between $R$ and its QF-subring $e R e$ which is the endomorphism ring of a minimal faithful $R$-module, and $R$ is uniquely determined up to isomorphism by $e R e$ and the numerical invariants $\delta_{k}(k=1, \ldots, m)$. For example, using the notation of Theorem 3.3 let $n=5, m=2, \quad l_{1}=2, \quad l_{2}=3$ and $T$ is Morita equivalent to an indecomposable, basic serial QF-ring $S=S_{1}+S_{2}+S_{12}+S_{21}$ where $S_{1}=e_{1} S e_{1}, \quad S_{2}=e_{2} S e_{2}, S_{12}=e_{1} S e_{2}, S_{21}=e_{2} S e_{1}, \quad 1=e_{1}+e_{2}$ with primitive orthogonal idempotents $e_{1}, e_{2}$ and the radicals $M_{1}, M_{2}$ of $S_{1}, S_{2}$, respectively, then $R$ is isomorphic to the generalized matrix ring

$$
\left(\begin{array}{ccccc}
S_{1} & S_{1} & S_{12} & S_{12} & S_{12} \\
M_{1} & S_{1} & S_{12} & S_{12} & S_{12} \\
S_{21} & S_{21} & S_{2} & S_{2} & S_{2} \\
S_{21} & S_{21} & M_{2} & S_{2} & S_{2} \\
S_{21} & S_{21} & M_{2} & M_{2} & S_{2}
\end{array}\right)
$$

and the QF-subring $e$ Re which is the endomorphism ring of the minimal faithful $R$-module, is isomorphic to $S$.

In the general case when $B$ is not necessarily zero, we have

Proposition 3.4. $A$ is a serial ring.

Proof. If $t=\varepsilon_{i} t \varepsilon_{j} \in B(i, j=1, \ldots, n)$, then $t$ maps $I_{i}$ in $I_{j}$ and $I t$ cannot contain $P_{j}$ because $P_{i} t=0$ by assumption. Hence $I_{i} t \subseteq P_{j} \subseteq R$. Therefore $I t \subseteq R$ for every $t \in B$ as $t=\left(\sum_{i=1}^{n} \varepsilon_{i}\right) t\left(\sum_{i=1}^{n} \varepsilon_{i}\right)$. Consequently, $B^{2}=0$. Since $B \triangleleft A$ and $A / B \cong R, A$ is a semiprimary ring. In view of Proposition 2.1 it is enough to see $\varepsilon_{i} t \varepsilon_{j} \in J(A)^{2}$ for each $t \in B$. Let $b=\varepsilon_{i} t \varepsilon_{j}$ and assume $b \neq 0$, then $P_{i} \neq I_{i}$, i.e., $P_{i}$ is not injective. Since $I_{i}$ is also projective, every submodule between $P_{i}$ and $I_{i}$ is projective. Thus without loss of generality one can assume $P_{i}=\operatorname{Ker}(t)$. Hence the socle of $P_{j}$ is contained in $J$ and isomorphic to $S_{[i+1]}$. Consequently there is a nonzero element $r=e_{[i+1]} r e_{j}$ in the socle of $P_{j}$ satisfying $e_{[i+1]} r=r$. 
Let $u=\varepsilon_{i} u \varepsilon_{[i+1]}$ and $v=\varepsilon_{[i+1]} v \varepsilon_{j}$ in $T$ extend $a_{i}$ and $r$, respectively. Then we have $\operatorname{Ker}(t)=\operatorname{Ker}(u v)$, i.e., $b \in J(A)^{2}$.

Since endomorphisms of $I_{i}$ send $P_{i}$ into itself, $\varepsilon_{i} A \varepsilon_{i}$ is a factor ring of $\varepsilon_{i} T \varepsilon_{i}$ for $i=$ $1,2, \ldots, n$. The equalities $\varepsilon_{l_{k}} T \varepsilon_{l_{k}}=\operatorname{End}\left(P_{l_{k}}\right)=\varepsilon_{l_{k}} A \varepsilon_{l_{k}}(k=1, \ldots, m)$ imply that the maximums of the lengths $c\left(R_{i} R_{i}\right)$ and $c\left(\varepsilon_{i} A \varepsilon_{i} \varepsilon_{i} A \varepsilon_{i}\right)(i=1, \ldots, n)$, respectively, are equal, say, to a constant $d$. Therefore both the lengths of ${ }_{R} R$ and ${ }_{A} A$ are at most $d n^{2}$. Moreover, being a factor ring of $A$, one obtains $c\left({ }_{R} R\right) \leq c\left({ }_{A} A\right)$. Let $R=A_{0}$. For $i>0$ let $T_{i}$ be the endomorphism ring of the injective module $I\left(A_{i-1} A_{i-1}\right)$, and $B_{i}, A_{i}$ the subsets of endomorphisms in $T_{i}$ sending $A_{i-1}$ into 0 and itself, respectively. Then $B_{i}$ is an ideal in $A_{i}$ and the factor ring $A_{i} / B_{i} \cong A_{i-1}$. Since $c\left({ }_{A_{i-1}} A_{i-1}\right) \leq c\left({ }_{A_{i}} A_{i}\right) \leq d n^{2}$ by the previous observation, after finitely many steps, say, $N$ we obtain a QF-ring $T_{N}$ which is the maximal quotien ring of $A_{N}$ and $R$ is a factor ring of $A_{N}$. One can now contruct $A_{N}$ from the serial QF-ring $T_{N}$ in the way suggested in Theorem 3.3. Thus we obtain as a final result

Theorem 3.5. Every basic indecomposable serial ring $R$ is a factor ring of an indecomposable basic serial ring whose maximal quotient ring is a QF-ring.

Example 3.0.1. If $Q$ is the factor of the path algebra of the quiver $\tilde{A}_{2}$ with arrows $a_{i}$ from $i$ to $[i+1](i=1,2,3 ; n=3)$ by the ideal generated by all paths of length 4 , then $Q$ is a weakly symmetric serial QF-ring. Let $R$ be the factor of $Q$ by the ideal generated by $a_{3} a_{2} a_{1}$ and $P_{i}=R e_{i}$ where $e_{i}$ is the idempotent associated to the vertex $i$. Then $\operatorname{Soc}\left(P_{1}\right) \cong S_{3}$ and $\operatorname{Soc}\left(P_{i}\right) \cong S_{i}$ for $i=2,3$ where $S_{i}$ is the simple module associated to $i$. Moreover, $I=I(R)=I\left(P_{1}\right) \oplus P_{2} \oplus P_{3}$ and $I\left(P_{1}\right) \cong P_{3}$. Therefore, $B$ is generated by any $\gamma=\varepsilon_{1} \gamma \varepsilon_{1}: I \longrightarrow I_{1}$ satisfying $\operatorname{Ker} \gamma=P_{1} \oplus P_{2} \oplus P_{3}$, and $A$ is a subring of $T=\operatorname{End}\left({ }_{R} I\right)$ isomorphic to $Q$.

Example 3.0.2. If $Q$ is the factor of the path algebra of $\tilde{A}_{2}$ by the ideal generated by all paths of length 6 , then although $Q$ is a serial QF-ring, $Q$ is not weakly symmetric. Let $R$ be the factor of $Q$ by the ideal generated by $a_{2} a_{1} a_{3} a_{2} a_{1}$, and 
put $P_{i}=R e_{i}$. Then $\operatorname{Soc}\left(P_{1}\right) \cong \operatorname{Soc}\left(P_{3}\right) \cong S_{2}$ and $\operatorname{Soc}\left(P_{2}\right) \cong S_{1}$. Furthermore, $I=I(R)=I\left(P_{1}\right) \oplus P_{2} \oplus P_{3}$ with $I\left(P_{1}\right) \cong P_{3}$ and $B=0$ hold.

Remark 3.0.5. Kupisch (cf. Folgerung 3.9 [11]) showed that every indecomposable (basic) serial ring $R$ satisfying $c_{i} \neq 1(\bmod n)(i=1, \ldots, n)$ is a factor of a QFring. The ring in Example 3.0.1 satisfies $c_{2}=c_{3}=1(\bmod n)$ and is a factor of a weakly symmetric serial QF-ring. However, by Example 3.0.2 there exists a factor of a serial QF-ring with $B=0$. Haack (cf. Example 4.7 in [5]) gave an example of a serial ring which is not a factor of any serial QF-ring.

As an application of Theorem 3.5 assume that $c_{1}=1$, i.e., $J e_{1}=0$ and $R$ is a serial ring with a simple projective module. Note that this condition is also satisfied by the rings $A_{i}$ constructed above as it is easy to check. Let now $R$ be a serial such that $P_{1}$ is simple and the maximal quotient ring of $R$ is a QF-ring, i.e., $B=0$. If $P_{l_{1}}$ is an indecomposable injective projective module with the socle isomorphic to $P_{1}$, then $a_{l_{1}}$ induces a projective cover $P_{l_{1}} \longrightarrow J\left(R e_{\left[l_{1}+1\right]}\right)$ with the kernel, say, $P_{i}, \quad i \neq l_{1}$ if $l_{1} \neq n$. Since all nonzero submodules of $P_{l_{1}}$ are projective, the condition $l_{1} \neq n$ implies that there exists a nonzero homomorphism from $I_{i}$ into $I_{\left[l_{1}+1\right]}$ sending $P_{i}$ to 0 , i.e., $B \neq 0$. This contradiction show that $l_{1}=n$ and hence the maximal quotient ring of $R$ is a matrix ring over a division ring, say $F$ and $R$ is an upper triangular matrix ring over $F$. Thus we reobtain the well-known result (cf. Theorem $32.7[3]$ )

Corollary 3.6. A basic indecomposable serial ring is a factor ring of a serial ring with projective socle if and only if it has a simple projective module. A basic indecomposable serial ring with projective socle is an upper triangular matrix ring over a division ring.

\section{WEAKLY SYMMETRIC SELFDUALITY OF SERIAL RINGS}

Recall that an artinian ring is locally distributive if the lattices of submodules of indecomposable projective left or right modules are distributive. We begin with a basic observation of Haack (cf. Proposition 4.1 [5]) 
Proposition 4.1. Every weakly symmetric selfduality of a locally distributive ring is a good duality.

Proof. Assume that ${ }_{R} E_{R}$ induces a weakly symmetric selfduality for a basic indecomposable locally distributive ring $R$. We have to show $K=r_{R} r_{E}(K)$ for every ideal $K$ of $R$. Let $1=e_{1}+\cdots+e_{n}$ be a decomposition of 1 as a sum of pairwise orthogonal primitive idempotents $e_{i}$ and $X=r_{R} r_{E}(K), R_{i}=e_{i} R e_{i}, E_{i}=e_{i} E e_{i}$. To complete the proof it is enough to see that $e_{i} K e_{j_{R_{j}}}$ and $e_{i} X e_{j_{R_{j}}}$ have the same length for all $i, j$ because the $R_{i} e_{i} R e_{j} R_{j}$ are uniserial on both sides of the same length in view of the local distributive condition. Since $E$ induces a weakly symmetric selfduality for $R$, the natural nondegenerate pairing $e_{i} R \times E e_{i} \longrightarrow E_{i}$ shows that $e_{i} R$ is the dual of $E e_{i}$ with respect to the selfduality of $R_{i}$ induced by $E_{i}$. Consequently, by putting $V_{i}=E e_{i}, W_{i}=r_{E}(K) e_{i}$ we have

$$
\begin{gathered}
c\left(e_{i} X e_{j_{R_{j}}}\right)=c\left(R_{j} e_{j}\left(V_{i} / W_{i}\right)\right)=c\left(R_{j} e_{j} V_{i}\right)-c\left(R_{j} e_{j} W_{i}\right)= \\
=c\left(e_{i} R e_{j_{R_{j}}}\right)-c\left(e_{i} R / K e_{j_{R_{j}}}\right)=c\left(e_{i} K e_{j_{R_{j}}}\right)
\end{gathered}
$$

from which the statement follows.

Now we are able to give a conceptual proof to a well-known result (cf. [4], [16]).

Theorem 4.2. Every serial ring admits a weakly symmetric selfduality.

Proof. Using the notation of Sections 2 and 3, in view of Proposition 4.1, Proposition 2.2 and Theorem 3.5 one can assume that $R$ is a basic indecomposable not selfinjective ring with $B=0$. By Proposition 3.1 $T=\operatorname{End}\left({ }_{R} I\right)=\operatorname{End}(I(R))$ is a QF-ring and one can identify $\varepsilon_{i}(i=1, \ldots, n)$ with $e_{i}$. For simplicity let $g_{k}=e_{l_{k}}(k=1, \ldots, m)$. There are two cases.

1. $T$ is weakly symmetric. Since the top and the socle of ${ }_{T} T g_{k}(k=1, \ldots, m)$ are isomorphic, they are such as $R$-modules, too, and hence with composition factors, starting from the bottom, $S_{\left[l_{\{k-1\}}+1\right]}, \ldots, S_{l_{k}}$. Consequently, if $M_{\left[l_{\{k-1\}}+1\right]}, \ldots, M_{l_{k}}$ are $R$-submodules of $P_{\left[l_{\{k-1\}}+1\right]}, \ldots, P_{l_{k}}$ of lengths $0,1, \ldots, \delta_{k}-1$, then the factor $R$ modules $I_{i} / M_{i}$ are injective with socle isomorphic to $S_{i}$. Otherwise $P_{\left[l_{k}+1\right]}$ would 
be an epimorphic image of the minimal submodule in $I\left(I_{i} / M_{i}\right)$ containing $I_{i} / M_{i}$, a contradiction. Since $M=\bigoplus_{i=1}^{n} M_{i}$ is a subbimodule of ${ }_{R} T_{R}$ (as it is easy to check in view of Theorem 3.3), the bimodule ${ }_{R} E_{R}=T / M=\bigoplus_{i=1}^{n} T \varepsilon_{i} / M_{i} \cong$ $\bigoplus_{i=1}^{n} I\left(P_{i}\right) / M_{i}$ induces a weakly symmetric selfduality for $R$.

2. $T$ is not weakly symmetric. By Proposition 2.2 there is an automorphism $\Psi$ of $e T e=e R e$ fixing the set $\left\{g_{k} \mid k=1, \ldots, m\right\}$ such that an $e R e-e R e$-bimodule $e e_{\Psi}:=e$ Re defined by $a * x * b=a x \Psi(b) \quad(a, x, b \in e R e)$ induces a weakly symmetric selfduality for $e R e$. Moreover, if $T e_{\Psi}:=T e$ is an $T-e R e$-bimodule defined by $a * x * b=a x \Psi(b)(a \in T, x \in T e, b \in e R e)$, then

$$
T e_{\Psi} \underset{e R e}{\otimes}-: e R e-\operatorname{Mod} \longrightarrow T-\operatorname{Mod}
$$

is an equivalence functor and $V:=T e_{\Psi} \underset{e R e}{\otimes} e T$ is an $T-T$-bimodule. Since the socle of $e T g_{k}=e R g_{k}(k=1, \ldots, m)$ is isomorphic to $e R \Psi^{-1}\left(g_{k}\right) / e J \Psi^{-1}\left(g_{k}\right)$, it has an element $0 \neq x=\Psi^{-1}\left(g_{k}\right) x$. Therefore

$$
0 \neq e \otimes x=e \otimes \Psi^{-1}\left(g_{k}\right) x=e * \Psi^{-1}\left(g_{k}\right) \otimes x=e \Psi\left(\Psi^{-1}\left(g_{k}\right)\right) \otimes x=g_{k} \otimes x \in V g_{k}
$$

and hence the socle of $T_{T} V g_{k}$ is isomorphic to the top of $T g_{k}$. Therefore $T g_{k} / J(T) g_{k}$ is the socle of ${ }_{T} V e_{i}$ for all $i=\left[l_{\{k-1\}}+1\right], \ldots, l_{k}$. Thus, as in Case 1, their composition factors as left $R$-modules, starting from the bottom, are $S_{\left[l_{\{k-1\}}+1\right.}, \ldots, S_{l_{k}}$ and, if $M_{\left[l_{\{k-1\}}+1\right]}, \ldots, M_{l_{k}}$ are $R$-submodules of $V e_{\left[l_{\{k-1\}}+1\right]}, \ldots, V e_{l_{k}}$ of lengths $0,1, \ldots, \delta_{l_{k}}-1$, then $V e_{i} / M_{i}$ are injective $R$-modules having the socle isomorphic to $S_{i}$, respectively. Since $M=\bigoplus_{i=1}^{n} M_{i}$ is an $R-R$-subbimodule of $V$ as it is easy to check in view of Theorem 3.3, the $R-R$-bimodule $E=V / M$ induces a weakly symmetric selfduality for $R$.

\section{REFERENCES}

[1] I. K. Amdal et F. Ringdal, Catégories unisérielles, C.R. Acad. Sci. Paris, Série A, 267 (1968), 85-87.

[2] I. K. Amdal et F. Ringdal, Catégories unisérielles, C.R. Acad. Sci. Paris, Série A, 267 (1968), 247-249. 
[3] F. W. Anderson and K. R. Fuller, Rings and categories of modules, 2nd revised ed., Springer Verlag, Berlin-New York 1992.

[4] F. Dischinger and W. Müller, Einreihig zerlegbare artinsche Ringe sind selbstdual, Arch. Math. 43 (1984), 132-136

[5] J. K. Haack, Selfduality and serial rings, J. Algebra 59 (1979), 345-363.

[6] G. Ivanov, Character modules and endomorphism rings of modules over artinian serial rings, Abelian groups, rings and modules (Perth, 2000), 177-189, Contemp. Math. 273, AMS, Providence, RI 2001.

[7] J. Kado, The maximal quotient rings of left H-rings, Osaka J. Math. 27 (1990), 247-252.

[8] J. Kado and K. Oshiro, Selfduality and Harada rings, J. Algebra 211 (1999), 384-408.

[9] K. Koike, Examples of QF-rings without Nakayama automorphism and H-rings without selfduality, J. Algebra 241 (2001), 731-744.

[10] H. Kupisch, Über eine Klasse von Ringen mit Minimalbedingung I, Arch. Math. 17 (1966), 20-35.

[11] H. Kupisch, Über eine Klasse von Artinringen II, Arch. Math. 26 (1975), 23-35.

[12] J. Lambek, Lectures on rings and modules, Blaisdell Publ. Com., Waltham MA 1966.

[13] K. Oshiro, Structure of Nakayama rings, Proc. 20th Symp. Ring Theory (Okayama 1987), 109-133, Okayama Univ., Okayama, 1987.

[14] K. Oshiro, Theories of Harada in artinian rings and applications to classical artinian rings, Intern. Symp. Ring Theory, (Kyongju, 1999), 279-301, Trends Math., Birkhäuser Boston, Boston, MA, 2001.

[15] A. Rosenthal, Complete noetherian serial rings have a selfduality, Comm. Algebra 27(4) (1999), 4961-4967.

[16] J. Waschbüsch, Selfduality of serial rings, Comm. Algebra 14(4) (1986), 581-589.

Alfréd Rényi Mathematical Institute, Hungarian Academy of Sciences, H-1364 BuDAPEST PF.127, HUNGARY

E-mail address: anh@renyi.hu 